\title{
REVIEW
}

Received 00th January 20xx, Accepted 00th January 20xx

DOI: $10.1039 / x 0 \times x 00000 x$

\section{Polymerisation-Induced Self-Assembly (PISA) as a Straightforward Formulation Strategy for Stimuli-Responsive Drug Delivery Systems and Biomaterials: Recent Advances}

\begin{abstract}
Hien Phan, ${ }^{\mathrm{a}}$ Vincenzo Taresco, ${ }^{\mathrm{b}}$ Jacques Penelle, ${ }^{\mathrm{a}}$ Benoit Couturaud ${ }^{* a}$
ABSTRACT: In the interest of nanomedicine designs toward the ultimate clinical relevance, stimuli-responsive amphiphilic block copolymers have emerged as promising nanocarriers for enhancing site-specific and on-demand drug release in response to a range of stimuli such as $\mathrm{pH}$, the presence of redox agents, or temperature. The formulation of amphiphilic block copolymers into polymeric drug-loaded nanoparticles is typically achieved by various methods (e.g., oil-in-water emulsion solvent evaporation, solid dispersion, microphase separation, dialysis or microfluidic). Despite the several progresses that have been made, there remain many challenges to overcome in order to produce reliable polymeric systems. The main weakness of the above methods is that they imply very low solid contents $(<1$ wt\%) and multiple-step procedures, thus limiting their scope. Recently, a new self-assembly methodology, polymerisation-induced self-assembly (PISA), has shown great promise in the production of polymer-derived particles in a straightforward one-pot approach, whilst facilitating high yield, scalability, and cost-effectiveness with respect to pharmaceutical industry protocols. We therefore focus this review primarily on the most recent studies dealing with the design and preparation of PISAgenerated nano-objects that are responsive to specific stimuli, thus providing an insight into how PISA may become an effective formulation strategy for the preparation of precisely tailored drug delivery systems and biomaterials, while some of the current challenges, conundrum and limitations are also critically discussed.
\end{abstract}

\section{Introduction}

The highly complex behaviour of diseases demands that drug delivery systems and biomaterials be designed in such a way that they can respond to various pathogenic environments in an opportune and judicious manner. To improve therapeutic reliability, it is of utmost importance for particles to exhibit high drug loading and site-specific delivery, while efficiently favouring drug release in the diseased body areas. ${ }^{1,2}$ In this regard, functionalised materials displaying such tailored features have rapidly emerged as a promising toolbox for the design of many advanced drug delivery systems. ${ }^{3,4}$ The functionalisation can be achieved via the encoding of stimuli-sensitive functionalities into molecular structures, thus endowing on-demand responses to endogenous factors such as $\mathrm{pH}$, oxidation, and reduction, or to exogenous triggers such as photoirradiation, temperature, and ultrasound activation. ${ }^{5-11}$ On the other hand, synthetic polymeric nanomaterials are of particular interest as their colloidal properties and surface chemistry can be readily tuned to precisely meet the demand of individual applications. ${ }^{12-14}$ Altogether, stimuliresponsive polymer-based nanocarriers have gained rapidly

\footnotetext{
a. Institut de Chimie et des Matériaux Paris-Est (ICMPE), UMR CNRS 7182, 2-8 rue Henri Dunant, 94320 Thiais, France

${ }^{b .}$ School of Chemistry, University of Nottingham, University Park, Nottingham, NG7 2RD, UK

See DOI: $10.1039 / \times 0 \times x 00000 x$
}

growing research attention in the biomedical sciences as a versatile and exciting platform for materials design strategies. ${ }^{15-20}$ However, polymer synthesis and formulation into polymeric nanoparticles (NPs) usually

involves multiple-step procedures, addition of toxic surfactants, and extensive intermediate purifications. In an attempt to minimise these tedious synthetic efforts whilst maintaining batch-to-batch consistency and clinical relevance, amphiphilic block copolymers have been adopted as nano-vehicle materials since they can inherently self-assemble in aqueous media to form stabilised coreshell nano-objects. ${ }^{21,22}$ Amphiphilic diblock copolymers have therefore been widely exploited in a range of therapeutic purposes, including cancer ${ }^{23,24}$ and gene therapy, ${ }^{25}$ and also in diagnosis. ${ }^{26}$

Typically, the room temperature self-assembly of amphiphilic block copolymers with a glassy hydrophobic block is usually obtained using the co-solvent or solvent switch method. ${ }^{27}$ In this technique, the amphiphilic block copolymer is initially dissolved in an organic solvent such as DMF, acetone, or THF that is compatible with both blocks, followed by the slow addition of a selective solvent, e.g. water, which is a good solvent for only one block, leading to the microphase separation and then to spontaneous self-assembly into nano-objects with a solvophobic core coated by a solvophilic layer. ${ }^{22}$ This allows for relatively low polymer concentration $(<1$ 
wt\%) to be used. ${ }^{28-31}$ In addition, the self-assembly usually occurs via post-polymerisation from well-defined precursor copolymers, and hence is not economically ideal for scale-up and industrial environments. While the solution-phase self-assembly can still leverage a diverse set of particle morphologies such as micelles, nanofibers, or vesicles, ${ }^{32,33}$ primarily dictated by the packing parameter $(p),{ }^{27}$ in practice it is tedious to obtain higher order morphologies.

To meet such technical challenges, polymerisation-induced selfassembly (PISA) has recently been proposed. PISA has tremendously redefined the concept of block copolymer self-assembly by jointly facilitating polymerisation and self-assembly in an one-pot approach. $^{34-41}$ Two types of polymerisations are used in PISA, dispersion and emulsion polymerisations. The former requires both monomers and a precursor macro-CTA to be solvophilic and compatible with each other (co-miscible ?) in a continuous phase, either in an organic solvent or water-based systems. Once a critical degree of polymerisation of the core-forming monomer is reached, this block becomes insoluble and tends to precipitate. The initial particles act then as the loci for the polymerisation, with monomers continuing to add covalently to the stabilised particles. Meanwhile, the second method is solely implemented in water, using a soluble homopolymer precursor that is chain-extended with an immiscible monomer to a critical degree of polymerisation. This leads to the formation of monomer droplets stabilised by the initial homopolymers. Both polymerisation methods generate in situ selfassembled nano-objects with various morphologies as depicted in Fig 1 , which is a very important and valuable asset compared to the typical nanoprecipitation technique in which mostly spherical particles are produced while the same copolymers by PISA can be shaped in several ways. ${ }^{42}$ The morphological evolution initially starts with the early formation of spherical micelles and provides a not-kinetically-frozen structure that evolves to cylindrical or wormlike nanoparticles, and finally to vesicles over the growing course of the core-forming block. The control over particle size and shape has been thoroughly discussed in recent reviews. ${ }^{43-50}$ Generally, the formation and transformation of NPs morphologies are controlled and predictable through which higher order sphere-to-vesicle transition is promoted with the increasing degree of polymerisation (DP) and reaction conditions. This feature facilitates the precise production of targeted particle shapes at ease. In general, controlling particle shape is of substantial importance to the performances of drug delivery systems as morphologies have each their own effects on the in vivo fate, biodistribution and cellular uptake after administration. ${ }^{51-53}$ More specifically, oblate particles exhibit a reduced uptake by macrophages and a prolonged blood circulation, strongly enhancing the odds of reaching the targeted sites. Due to their high surface areas, elongated NPs can also form stronger ligand-receptor bindings in active targeting over nanospheres, and thus improve the selectivity and specificity during endocytosis. ${ }^{54-56}$ On the other hand, NPs with sharp edges can disrupt the endosomal membranes, inducing less exocytosis, and so stay longer in the cytoplasm than spherical particles. ${ }^{57}$ By producing a wide scope of well-controlled nanostructures, PISA can provide more possibilities for achieving a better cellular uptake and tracking, ${ }^{58}$ which ultimately results in an enhanced bioavailability of the NPs. NP uptake at target cells are also directly governed by their sizes. NPs should ideally be designed with sizes between 5 and 100 $\mathrm{nm}$ in diameter. Sizes lower than $5 \mathrm{~nm}$ are known to generate particles that will be eliminated by renal filtration, ${ }^{54}$ whereas higher diameters (over $200 \mathrm{~nm}$ ) trigger excessive accumulation in the liver and spleen, ${ }^{59}$ while particles with diameters lower than $100 \mathrm{~nm}$ exhibit less adsorption of serum proteins while circulating in the blood, thus enhancing half-lives in vivo. ${ }^{60,61}$ Moreover, PISA can be implemented at relatively high solid contents up to $50 \mathrm{wt} \%$ and usually imparts very high monomer conversions within short reaction times. Hence, PISA is of high benefit to the NPs formulation, with tuneable shapes and sizes available even during large-scale productions. Besides, the simultaneous self-assembly and drug encapsulation may enable relatively high loading efficiencies $\left(67 \%\right.$ for doxorubicin-encapsulated NPs ${ }^{62}$ vs. $54 \%$ for cisplatin-loaded micelles or $24 \%$ for BSA-loaded vesicles, ${ }^{63}$ under simple working conditions), likely owing to high polymer concentrations. PISA synthesis have been carried out under several types of living polymerisation techniques, but reversible addition-fragmentation chain transfer (RAFT) is probably the most prevalent synthetic method currently available to prepare NPs by a PISA approach due to its adaptability to a broad range of reaction conditions and monomer families. ${ }^{64-69}$ RAFT dispersion or emulsion PISA processes can be initiated via various approaches, most commonly by thermal initiation but also by other new alternative approaches such as under photochemical, ultrasound and microwave activations, or using enzymes. ${ }^{50}$ Moreover, a few emerging types of polymerisations have recently emerged that can be of interest to PISA for drug delivery formulations, e.g., ring opening polymerisation (ROP) ${ }^{70}$ or ring-opening metathesis polymerisation PISA (ROMPISA). ${ }^{71}$ In conclusion, with superior cellular uptakes resulting from diverse and tuneable morphologies as well as relatively high drug loading under industrially relevant approaches, PISA appears as a versatile synthetic and formulative technique for polymer-based nanomaterials in drug delivery strategies.

Based on simple and accessible formulations of NPs from PISA, an extensive amount of literature has been focused on producing stimuli-sensitive NPs, using mostly RAFT and some ROP/ROMP combination, thereby conferring an efficient programmable drug release through a timely and direct nano-formulation. The present review therefore aims at highlighting a selected number of the latest exciting examples in the design and preparation of polymeric NPs sensitive to a variety of stimuli triggers by mainly RAFT-PISA and a few ROP/ROMPISA that show remarkable applications on many precision drug delivery systems. The authors will conclude with several critical remarks on the challenges and opportunities underlying the role of PISA as a formulation strategy for polymerbased nanomedicine in the context of clinical translatability.

\section{Summary of the several stimuli used for drug delivery systems as obtained by PISA}

\section{1. pH-Responsive systems}

Difference in $\mathrm{pH}$ profiles under pathogenic conditions compared to normal cell microenvironments are one of the most prominent 
features to exploit in the development of intelligent tools in nanomedicine. Many inflammation sites and solid tumours display relatively acidic $\mathrm{pHs}(<6.5)$ compared to normal tissues $(\mathrm{pH} 7.4) .{ }^{73,74}$ Integrating functionalities into particles that are stable under physiological $\mathrm{pH}$ whilst cleavable at low $\mathrm{pH}$ thus allows to trigger drug unloading in a well-programmed way.

For example, Karagoz et al. described a one-pot PISA synthetic route to asymmetric-block-copolymer NPs: poly[oligo(ethyleneglycol)methacrylate]-block-[poly(styrene)-co-

poly(vinyl benzaldehyde)] (POEGMA- $b$-P(ST-Co-VBA)) was obtained by these authors using a RAFT-mediated dispersion polymerisation in methanol and enabling self-assembly into various morphologies from spheres, worm-like and rod-like to vesicles. ${ }^{75}$ After the particles synthesis, the aldehyde groups in the core-forming blocks were conjugated with doxorubicin (DOX) via its amine functional groups to form $\mathrm{pH}$-responsive imine moieties $(-\mathrm{C}=\mathrm{N}-)$ on Schiff bases. A release study demonstrated that more than $80 \%$ DOX was liberated from the NPs at $\mathrm{pH} 5$ due to the acidic $\mathrm{pH}$-breakable bonds, whereas only $10 \%$ were released at $\mathrm{pH}$ 7.4. Furthermore, while particle morphologies did not affect the DOX release, they had a significant impact on the cell internalisation in MCF-7 breast cancer cell line: worm- and rod-like particles displayed an improved cell internalisation compared to spherical micelles and vesicles. The authors reasoned that cylindrical morphologies imparted greater aspect ratios or surface areas, allowing for higher adhesions to cell membranes and thus favouring cell uptake. In addition, rod-like and worm-like DOX-particles exhibited higher cytotoxicities than vesicles and micelles, as observed from IC $\mathrm{C}_{50}$ variations on a MCF-7 cell line (Fig 2). In summary, this system among all possible obtained morphologies demonstrated an effective combination of responsiveness, cellular internalisation and cytotoxicity, a feature very difficult to achieve from a conventional nanoprecipitation.

Similarly, (methacryloxyethoxy)benzaldehyde (MAEBA) was polymerised in the presence of poly $\left(\left(\mathrm{N}_{\mathrm{N}} \mathrm{N}^{\prime}\right.\right.$-dimethylamino $)$ ethyl methacrylate) used as a macro-Chain Transfer Agent (macro-CTA) under RAFT dispersion PISA conditions, generating PDMAEMA block copolymer nano-objects as spheres, nanorods, nanowires and vesicles. ${ }^{76}$ DOX was conjugated with the PMAEBA block via the acidic $\mathrm{pH}$-breakable imine linkage (-C=N-) in a similar way as in Ref ${ }^{75}$. A drug release study indicated that less than $1 \%$ of the DOX had been released at $\mathrm{pH} 7.4$, while the fastest release was observed at $\mathrm{pH} 5$ due to the cleavage of the aromatic imine under acidic condition.

Lately, Arms and coworkers synthesised bio-inspired framboidal vesicles that simulated some Dengue virus features, with the vesicles exhibiting both moieties targeting the SR-B1 scavenger receptors that are available on triple-negative breast cancer cells, and $\mathrm{pH}$-sensitive surface areas for local drug delivery. ${ }^{77}$ In particular, two macro-CTAs poly(glycerol monomethacrylate) (PGMA) and poly(2-(methacryloyloxy)ethyl phosphorylcholine) (PMPC), were first chain-extended using 2-hydroxypropyl methacrylate (HPMA) and a GlyMA that had been conjugated with rhodamine B piperazine (Rh). The obtained PGMA-P(HPMA-statGlyMARh) polymer was then chain-extended with the 2(diisopropylamino)ethyl methacrylate (DPA) monomer via a RAFT

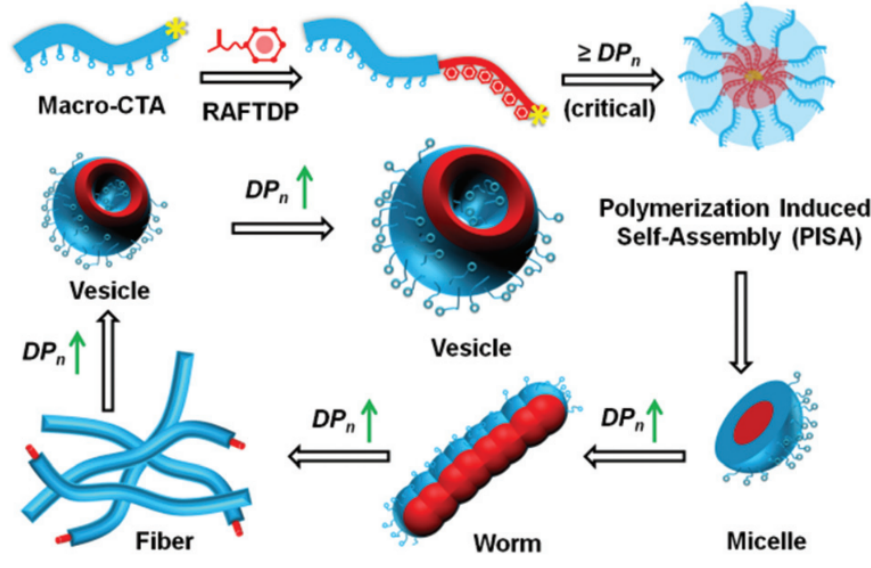

aqueous-seeded emulsion polymerisation, resulting in a $\mathrm{pH}$ responsive framboidal triblock copolymer vesicles. In this respect, the phosphorylcholine functionalities of PMPC endowed the vesicles with selective ligands to target the SR-B1 receptors available on MDA-MB-231 cells, while the tertiary amines on DPA became protonated to liberate the plasmid DNA in the nucleus

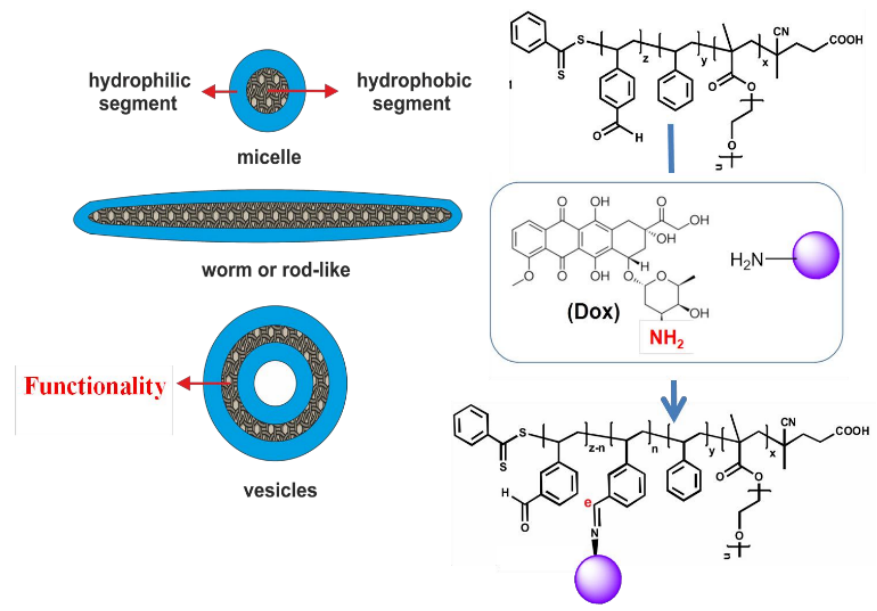

through intracellular acidification (Fig 3).

Another recent example was reported on the synthesis of cisplatin drug-encapsulated spherical micelles using ROMPISA. ${ }^{78}$ One of the most attractive features of ROMPISA is its tolerance with respect to a wide range of initiators and functionalities, allowing for the straightforward polymerisation of monomers in one pot. Amphiphilic block copolymers were synthesised in water, particularly a poly(oligo

Fig 1. Illustrative mechanism of NP morphological transformation by RAFT-PISA. Taken from Ref ${ }^{72}$ with permission from The Royal Society of Chemistry.

Fig 2. Schematic diagram of in situ self-assembly of POEGMA-b$\mathrm{P}(\mathrm{ST}$-co-VBA) using PISA and its conjugation with DOX through a $\mathrm{pH}$ cleavable bond. Reproduced from Ref ${ }^{75}$ with permission from The Royal Society of Chemistry.

ethylene glycol) macro CTA, which was chain-extended by comonomers cisplatin analogue norbornene dicarboximide and $\mathrm{pH}$ - 
(a)

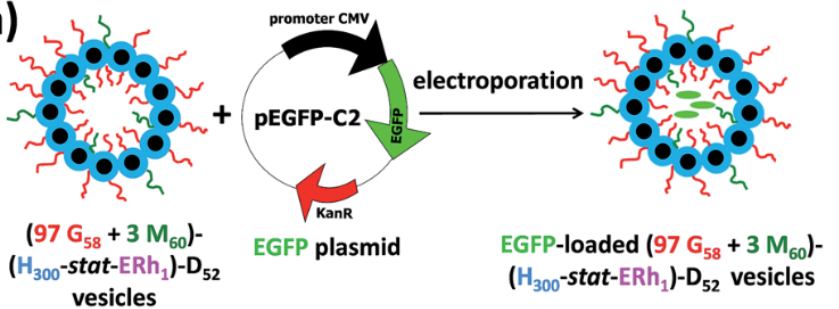

(b)

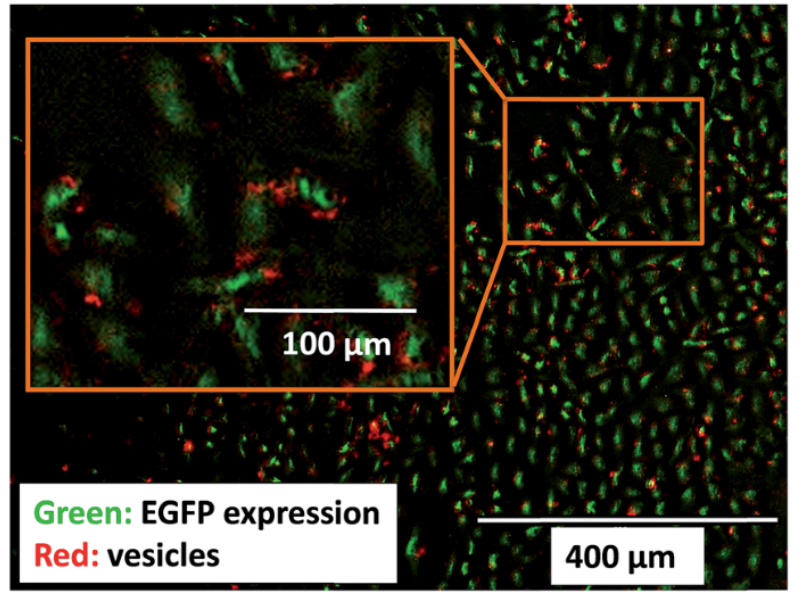

responsive 2-(diisopropylamino)ethyl norbornene dicarboximide. The effect of sizes and surface charges on the resulting micelles was investigated with respect to cell uptakes and cytotoxicities, indicating that smaller sizes exhibit improved cell uptakes and cytotoxicities, whilst positively-charged NPs were better absorbed than neutral or negatively-charged ones. A drug loading of $20 \%$ was achieved at $20 \%$, with a selective release at $\mathrm{pH} 6$.

\subsection{Reduction-responsive systems}

High intracellular redox potentials is another hallmark of carcinogenesis, resulting from an overproduction of reductive agents such as the glutathione tripeptide $\gamma$-glutamyl-cysteinylglycine (GSH), whose concentration ranges from 2 to $10 \mathrm{mM}$ in the cytosol of tumour cells

Fig 3. Illustration of the intracellular release of a EGFP plasmid DNA within MDA-MB-231 cells using 97 PGMA $_{58}+3$ PMPC $\left._{60}\right)-$ P(HPMA 300 -stat-GlyMARh 1$)-$ PDPA $_{52}\left(97 \mathrm{G}_{58}+3 \mathrm{M}_{60}\right)-\left(\mathrm{H}_{300}\right.$-stat$\left.E R h_{1}\right)-D_{52}$ framboidal vesicles. (a) Schematic diagram of the loading of plasmid EGFP DNA in these triblock copolymer vesicles (the green colour indicates a successful EGFP expression while the red colour indicates the location of the rhodamine-labeled vesicles). Reproduced from Ref ${ }^{77}$ with permission from The Royal Society of Chemistry.

compared to the 1-2 mM concentration typical of normal cells. It is thus widely adopted to facilitate targeted and on-demand drug releases. ${ }^{79-82}$ Reductive responsiveness is also critically important in hypoxia, a biological condition associated with oxygen deprivation in various diseases such as rheumatoid arthritis, ischemic stroke, and solid tumours. Hypoxia is associated with the presence of reductive species such as azoreductases and nitroreductases (NTR), thus nanomedicinal tools can be tailored using azobenzene (Azo), nitroaromatic or quinone derivatives as hypoxia-responsive functionalities that are likely cleavable within hypoxia-affected areas. $^{83,84}$

In this regard, anticancer drug camptothecin (CPT) was integrated in a prodrug monomer (CPTM). 2-(2-(Methoxyethoxy)ethyl methacrylate $\left(\mathrm{MEO}_{2} \mathrm{MA}\right)$ and the obtained CPTM comonomer were then polymerised in the presence of poly(ethylene glycol)-4-cyano4-((phenylcarbono-thioyl)thio) pentanoic acid ( $\left.\mathrm{PEG}_{113}-\mathrm{CPDB}\right)$ used as macro-CTA, via a RAFT dispersion technique, leading to the PEG$b$-P(MEO ${ }_{2}$ MA-co-CPTM) diblock prodrug copolymer. ${ }^{85}$ This synthetic technique resulted in prodrug NPs with a three-layered structure. In particular, the reduction-sensitive core was cross-linked via a copolymerisation of benzyl methacrylate (BzMA) and N,Ncystaminebismethacrylamide (CBMA); the second layer contained the CPT-copolymer with the CPT conjugated to the polymer chains via reductive-sensitive disulfide linkages; and the outer layer included stabilising hydrophilic PEG chains. This prodrug NPs facilitated the cleavage of the conjugated CPTs upon exposure to a reductive microenvironment, e.g. at high levels of GSH in the cytosol. The synthetic strategy enabled an intriguing drug delivery system achieving (1) a one-pot simultaneous polymerisation and drug encapsulation at a relatively high concentration of $100 \mathrm{mg} . \mathrm{mL}^{-}$ ${ }^{1}$, (2) a high monomer conversion, (3) a simultaneous core-crosslinking during prodrug particle preparation, (4) a CPT concentration that could be precisely tuned by altering the $\mathrm{MEO}_{2} \mathrm{MA} / \mathrm{CPT}$ ratio, and (5) an efficient drug unloading upon cell internalisation due to the high concentration of GSH in the cytoplasm.

Recently, the development of dual-functional polymer NPs exhibiting a fluorescence-assisted tracking capability and reductionresponsive triggered drug release was reported. ${ }^{86}$ Accordingly, azaboron dipyrromethene (BODIPY), a near-infrared (NIR) fluorescence dye, was loaded in situ into particles via a RAFT aqueous dispersion PISA without specific purifications. Firstly, a reduction-responsive polyprodrug camptothecin-monomer (pCPTM) was prepared via a chain extension of a PPEGMA macro-CTA during the RAFT copolymerisation of PEGMA and CPTM. Secondly, PEGMA comonomers and pre-synthesised NIR fluorescent monomer (NIRM) were co-polymerised in the presence of the PPEGMA macro-CTA, yielding $\mathrm{pNIRM}$. The last step was required to prepare core-crossedlinked bifunctional NPs, wherein PCPTM and PNIRM were hydrophilic macro-CTA agents chain-extended with the HPMA monomer and dimethacrylate cross-linker (EGDMA) in a one-pot RAFT aqueous dispersion polymerisation. The resulting spherical micelles had a three-layered structure, with a cross-linked core encapsulating the CPT drug, surrounded by the pNIRM fluorescent layer, and externally coated by the hydrophilic PPEGMA corona. The covalent attachment of BODIPY imaging agent endowed distinguished (specific, distinctive ?) optical properties to the spheres, enabling the real-time monitoring and tracking of the loaded drugs and NPs via an NIR imaging technique. In addition, both cell internalisation and intracellular drug release induced by a high level of GSH (10 mM) was compellingly confirmed in HeLa cells in vitro. In addition, the in vivo imaging clearly indicated a passive targeting of the spherical micelles in tumour site over time, with constantly visualised fluorescent signals. Taken as a whole, PISA has successfully allowed for the preparation of highly delicate spherical micelles with reductive-responsive drug release and stable optical characters via a direct and simple one-pot formulation strategy, and thus paving a novel pathway for tracking dynamic processes and biological imaging therapies (Fig 4). 
Couturaud et al. synthesised functionalized fluorine-based amphiphilic block copolymers using a photo-mediated RAFT dispersion polymerisation, as promising scaffolds for drug delivery. ${ }^{87}$ Due to the readily functionalisation with amino compounds, ester-activated pentafluorophenyl methacrylate (PFMA) was employed and polymerised with the poly(ethylene glycol)-4-cyano-4-(ethylthiocarbonothioylthio)pentanoic acid (PEG $113-$-CEPA) macro-CTA, via light-triggered PISA at $405 \mathrm{~nm}$ and at $37^{\circ} \mathrm{C}$, yielding uniform spherical micelles independently of given DP variations. A post-functionalisation with primary amines, e.g., ethylenediamine (EDA) or cysteamine, with the pentafluorophenyl ester groups generated amide cross-linking with the core-forming block, imparting a greater colloidal stability to the spherical particles. Upon exposure to increasing levels of GSH, the cysteamine-bearing nano-structures dissociated due to the reduction in GSH-sensitive disulfide cross-links, whereas the diamine-cross-linked spherical micelles remained intact. The designed cross-linked polymeric NPs also had excellent biocompatibilities with respect to in vitro human cells (Fig 5).

A recent work by Hong and coll. demonstrated dual reductiontriggered drug release and $\mathrm{pH}$-responsive charge-reversible characteristic of prodrug NPs prepared by PISA. ${ }^{88}$ Poly(N-(2hydroxypropyl)methacrylamide) (PHPMA) and poly(2(diethylamino)ethyl methacrylate) (PDEAEMA) were used as macro CTAs and chain-extended with CPTM to produce PHPMA/PDEAEMA-stabilized NPs with $10 \%$ of polymer concentration. The PDEAEMA chains were protonated in acidic tumours and transitioned from a hydrophobic to a hydrophilic state, which in turn enabled an increased drug release and cancer cell uptake.

\section{Oxidation-responsive systems}

While a moderate level of ROS (requiert une definition de l'abréviation) is a key necessity to maintain normal functions in cell signaling pathways and metabolism through oxygen-regulating homeostasis, a large increase in ROS is associated with a variety of pathological conditions such as cancer, diabetes and inflammations. ${ }^{89,90}$ Hence, the overexpression of ROS has been employed for the design of smart oxidation-responsive polymerbased nanomaterials. ${ }^{91-95}$

Having realised the current stimuli-sensitive nanocarriers usually require tedious preparations, Sobotta et al. developed a one-pot synthetic pathway using RAFT-mediated dispersion PISA to produce well-defined spherical micelles of tuneable sizes and displaying ROS-triggered drug release. ${ }^{96}$ A series of block copolymers with varying chain ratios was prepared, whereby poly $(\mathrm{N}-$ acryloylmorpholine) (PNAM) was utilised as a macro-CTA for chain extension with $\mathrm{N}$-acryloylthiomorpholine (NAT), a monomer carrying a oxidation-sensitive thioether moiety. This procedure readily afforded P(NAM-b-NAT) spherical particles of $25-73 \mathrm{~nm}$ in diameter (DLS) via the PISA process.

The sphere size-range could be tailored by tuning the size or composition of the diblock copolymers. Nile Red dye was chosen as a model for the drugs, and was encapsulated into the spheres. In response to a $\mathrm{H}_{2} \mathrm{O}_{2}$ concentration at $10 \mathrm{mM}$, the hydrophobic thioether was oxidised to the hydrophilic sulfone, triggering a solubility transition for the core-forming block. As a result, the micellar structures dissociated, and the drug was released as confirmed by a fluorescence quenching of Nile Red proportional to

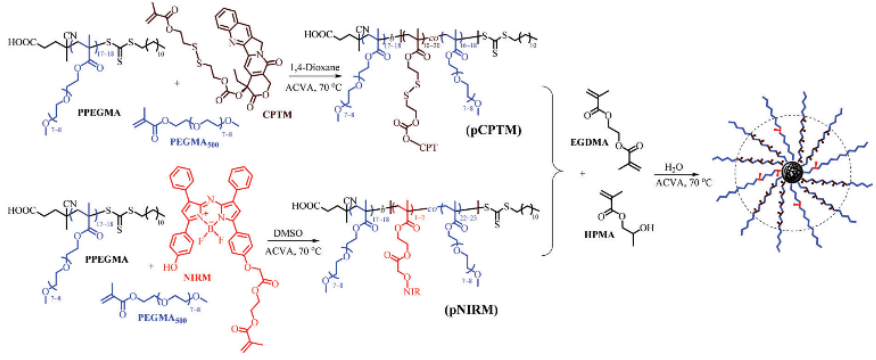

increased $\mathrm{H}_{2} \mathrm{O}_{2}$ levels. In addition to oxidation-triggered drug releases, the formulated nano-vehicle showed excellent biocompatibilities and no cytotoxicities, therefore providing a versatile and exciting avenue towards selective and controlled drug deliveries to sites of interests (Fig 6).

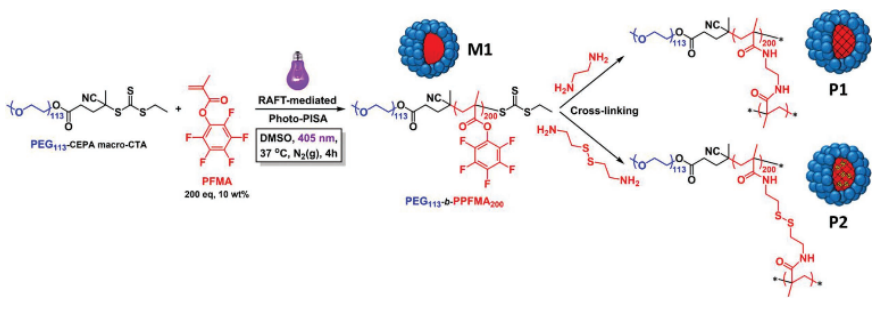

\subsection{Carbon dioxide $\left(\mathrm{CO}_{2}\right)$-responsive systems}

Unlike other experimental models for stimuli that usually involve chemical stimulations and possibly generate by-products that can have side effects on biological cells, $\mathrm{CO}_{2}$ appears as an attractive alternate stimulus since it is a key endogenous metabolite with mild

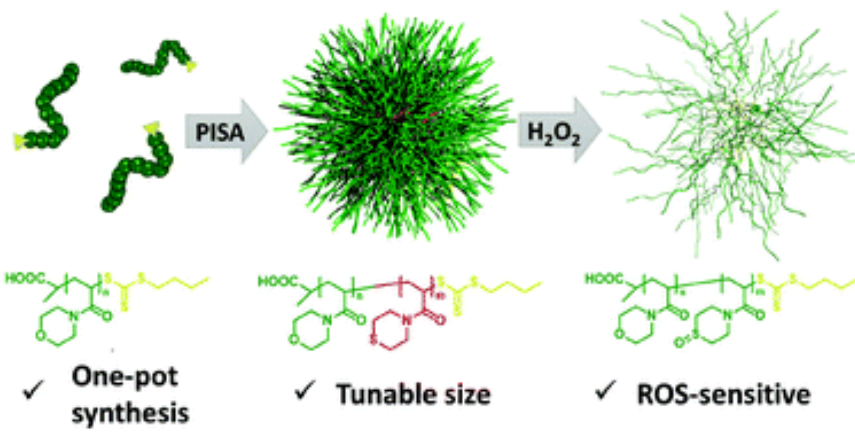

and green nature. Additionally, $\mathrm{CO}_{2}$ exhibits excellent biocompatibility and membrane permeability. ${ }^{97-101}$ Even though $\mathrm{CO}_{2}$ responsiveness has not been largely adopted in the context of therapeutic treatments, some of the designs available for $\mathrm{CO}_{2}$ sensitive polymeric materials have been considered as proof-ofconcepts for uses in drug delivery.

Recently, silica nanoparticles $\left(\mathrm{SiO}_{2} \mathrm{NPs}\right)$ have been utilised as effective adhesives for hydrogels and biological tissues. ${ }^{102,103}$ It was reported that $\mathrm{SiO}_{2} \mathrm{NPs}(20 \mathrm{~nm})$ can be encapsulated into PISAproduced hybrid vesicles displaying an on-demand release of the internal contents due to the $\mathrm{CO}_{2}$-responsive character of the coreforming block. Here, $\mathrm{mPEG}_{113}$ was exploited as a macro-CTA for the photo-initiated RAFT

Fig 4. Synthetic routes for reductive-responsive, prodrug and NIRfluorescence imaging dual-functional NPs by RAFT mediated dispersion polymerisation, producing P(CPTM-co-NIRM)-b-P(HPMACo-EDGMA) spherical micelles in water. Reproduced from Ref ${ }^{86}$ with permission from The Royal Society of Chemistry. 


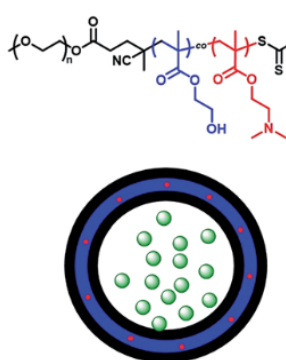

Hybrid Vesicles

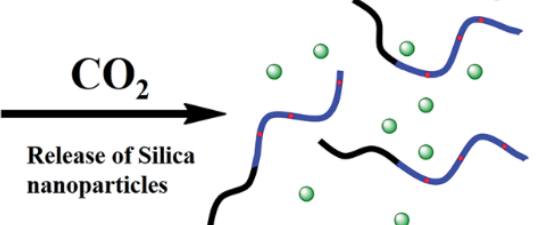

Dissolved copolymer chains + free silica nanoparticles

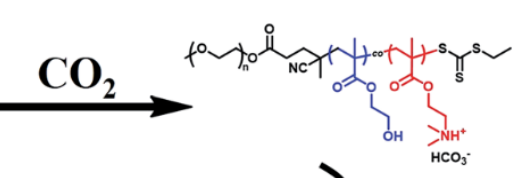

Fig 5. Schematic illustration of the preparation of PEG-b-PPFMA via photo-initiated PISA in DMSO at $37^{\circ} \mathrm{C}$ (M1), followed by crosslinking reactions using either ethylenediamine or cysteamine (P1 and $P 2$, respectively) for transfer into aqueous media. Reproduced from $\operatorname{Ref}^{87}$ with permission.

Fig 6. Schematic diagram of the one-pot synthesis of P(NAM-b-NAT) micelles of tuneable sizes displaying ROS-triggered drug release. Reproduced from Ref ${ }^{96}$ with permission from The Royal Society of Chemistry.

aqueous dispersion copolymerisation of 2-hydroxypropyl methacrylate (HPMA) and 2-(dimethylamino)ethyl methacrylate (DMAEMA) at room temperature. The obtained results indicated that complete monomer conversion was rapidly achieved within 15 min upon exposure to a $405 \mathrm{~nm}$ visible-light irradiation, affording a well-defined $\mathrm{CO}_{2}$-sensitive diblock copolymer mPEG- $b$-P(HPMA-coDMAEMA). The $\mathrm{SiO}_{2} \mathrm{NPs}$ were concurrently encapsulated into the lumen of the polymeric vesicles via PISA, providing a simultaneous and convenient one-pot synthetic strategy. The loaded cargos were released from the NPs in response to $\mathrm{CO}_{2}$ bubbling under mild conditions, due to the presence of the DMAEMA monomer in the hydrophobic block. After a 2 minutes treatment with $\mathrm{CO}_{2}$, DMAEMA was protonated, enhancing the hydrophilicity of the core-forming block, and the vesicles therefore disintegrated to release the $\mathrm{SiO}_{2}$ NPs (Fig 7). ${ }^{104}$ The same research group used the same concept

Fig 7. $\mathrm{CO}_{2}$-triggered release of $\mathrm{SiO}_{2} \mathrm{NPs}$ from mPEG-b-P(HPMA-coDMAEMA) hybrid vesicles. Reproduced from Ref ${ }^{105}$ with permission.

to encapsulate fragile biomacromolecules such as bovine serum albumin (BSA) (5 wt\%) into the polymeric vesicles prepared from an aqueous photo-initiated PISA under mild conditions. BSA was encapsulated in situ in hybrid vesicles made of PEGMA- $b$-P(HPMAco-DMAEMA). The addition of increased amounts of DMAEMA led to higher order morphologies during the block copolymer selfassembly, due to increased hydrogen bonding interactions. In the presence of $\mathrm{CO}_{2}, \mathrm{BSA}$ was unloaded from the vesicles due to the disassembly of copolymer chains induced by the protonation of DMAEMA. ${ }^{105}$

Nevertheless, several challenges remain to produce $\mathrm{CO}_{2}$-sensitive NPs via PISA. First, the polymerisation solution has to be judiciously degassed by purging inert gas or freeze-pump-thaw cycles, thus complicating a large-scale manufacturing of those NPs. Second, the obtained particles are likely to disintegrate upon exposure to surfactants or organic solvents. Third, the switchability of $\mathrm{CO}_{2}$ with

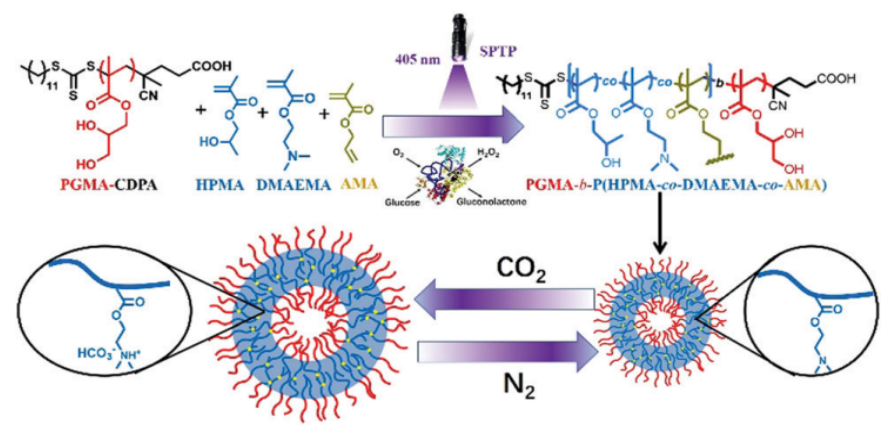

inert gas ( $\mathrm{N}_{2}$ or Argon) is limited. To answer these challenges, Yu et al. described a robust in situ cross-linking strategy that provides (1) a mild PISA process using photo-induced initiation in water; (2) an open-air polymerisation using enzyme-assisted degassing; (3) $\mathrm{CO}_{2-}$ responsive vesicles to precisely release the internal cargos. Particularly, HPMA and DMAEMA were polymerised, under RAFT aqueous dispersion copolymerisation, with 4-cyano-4(ethylthiocarbonothioylthio)pentanoic acid (CDPA) macro-CTA, to directly produce PGMA- $b$-P(HPMA-co-DMAEMA-co-AMA) vesicles via photo-induced PISA. ${ }^{106}$ The reaction was deoxygenated by adding glucose oxidase and glucose. In situ crosslinking was achieved by including allyl methacrylate (AMA), an asymmetric cross-linker, during the PISA process. Under $\mathrm{CO}_{2}$ treatment, the core-contained DMAEMA was protonated and became hydrophilic, in a similar way to the aforementioned studies. However, due to the AMA cross-linked core, the vesicles did not disrupt but became prone to swelling or expansion in volume and size. On the other hand, when purging with $\mathrm{N}_{2}$, the swelling vesicles shrunk because of the deprotonation of DMAEMA under these inert conditions. This swelling-shrinking process was reversible over four $\mathrm{CO}_{2} / \mathrm{N}_{2}$ cycles. Methylene Blue (MB) dye was used as a drug model in a proof-ofconcept release experiment, and revealed enhanced release when subjected to a $\mathrm{CO}_{2}$ environment. In the meantime, these $\mathrm{CO}_{2-}$ sensitive vesicles could carry an increased amount of $\mathrm{SiO}_{2} \mathrm{NPs}$ after the $\mathrm{CO}_{2}$ treatment. This was explained by the interactions between $\mathrm{SiO}_{2} \mathrm{NPs}$ with tertiary amines on DMAEMA in the hydrophobic block and the improved surface area of the vesicles favouring more $\mathrm{SiO}_{2}$ NPs to be loaded (Fig 8).

Fig 8. Schematic illustration of an enzyme-assisted photo-PISA for preparing cross-linked $\mathrm{CO}_{2}$-responsive vesicles. Reproduced from Ref ${ }^{106}$ with permission from The Royal Society of Chemistry.

\subsection{Temperature-responsive systems}

Typically, a temperature-sensitive block copolymer exhibits a lower critical solution temperature (LCST) at the interface between a stabilising block and a core-forming block, below which it is soluble and above which it becomes insoluble. A temperature oscillation around the LCST triggers a particle morphological transformation, leading to the disassembly of nano-structures and to the unloading of the internal payloads. This feature is interesting in the area of tissue engineering and regeneration as illustrated in the following examples.

Lately, Mable et al. prepared a series of in situ $\mathrm{SiO}_{2}$-encapsulated amphiphilic block copolymer vesicles via a one-pot RAFT aqueous dispersion polymerisation, in which a poly(glycerol monomethacrylate) (PGMA) macro-CTA was chain-extended with the HPMA monomer while adding simultaneously hydrophilic 18 
$\mathrm{nm} \mathrm{SiO} \mathrm{NPs}^{63} \mathrm{SiO}_{2} \mathrm{NPs}$ were utilised as a bio-related active species due to their intrinsic self-healing effects on synthetic hydrogels or biological tissues. ${ }^{107}$ The targeted degree of polymerization was maintained relatively low to explore the thermo-responsiveness of the PHPMA core-forming block. SAXS confirmed that the release of $\mathrm{SiO}_{2}$ NPs occurs within the first 12

min upon cooling to $0^{\circ} \mathrm{C}$, as the $\mathrm{PGMA}_{58}-b$ - $\mathrm{PHPMA}_{250}$ diblock copolymer underwent a morphological transformation from vesicles to a mixture of spherical and short-worm micelles, thereby triggering the release of internal cargos. Furthermore, an onset of vesicle dissociation was observed at about $10^{\circ} \mathrm{C}$, and promptly (regularly ?) increased as the temperature decreased. The authors reported this smart formulation and effective on-demand release of $\mathrm{SiO}_{2}$ NPs would be applied to self-healing adhesives of synthetic hydrogels and living tissues, thus enabling a possible model for encapsulating globular proteins (BSA), enzymes and antibodies (Fig 9).

On the other hand, worm-like gels have been widely adopted as potential materials in a range of biomedical applications. The group of Armes reported the preparation of biocompatible poly(glycerol monomethacrylate)-poly(2-hydroxypropyl methacrylate) (PGMA- $b$ PHPMA) diblock copolymer nanoworm gel that were responsive to temperature changes. ${ }^{108}$ These PGMA-b-PHPMA diblock copolymer worm-like micelles display a reversible thermal-responsive behaviour. Here, A PGMA- $b$-PHPMA diblock copolymer was dispersed into fluorophore-labelled Staphylococcus aureus at $4^{\circ} \mathrm{C}$, the temperature at which nanoworm degelation (gel deletion ?) occurred, and led to worm-to-sphere transition, thus lowering the solution viscosity. This solution was then filtered through a $0.45 \mu \mathrm{m}$ membrane, allowing for the smaller diblock copolymer particles to cross whereas the micrometer-sized bacteria stayed on the

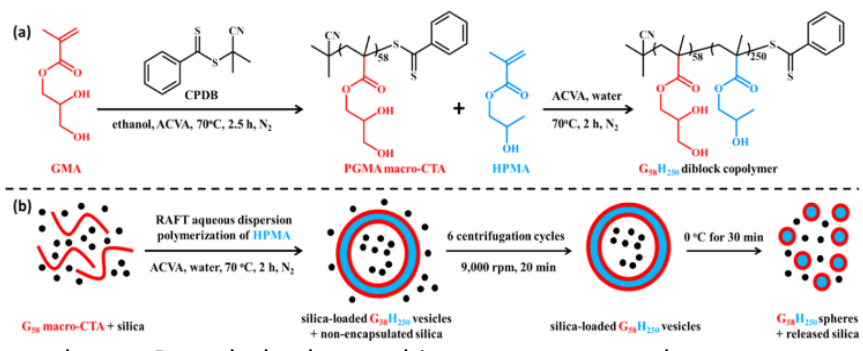

membrane. Brought back to ambient temperature, the

Fig 9. (a) Synthesis of $\mathrm{PGMA}_{58}-b-\mathrm{PHPMA}_{250}$ (G58H250) diblock copolymer via RAFT aqueous dispersion; (b) Schematic illustration of the

in situ encapsulation of $\mathrm{SiO}_{2} \mathrm{NPs}$ via PISA and subsequent release of $\mathrm{SiO}_{2} \mathrm{NPs}$ on cooling to $0{ }^{\circ} \mathrm{C}$ due to vesicle dissociation. Reproduced from $\operatorname{Ref}^{63}$ with permission.

obtained solution reversibly shifted from spheres to worms, favouring the sterilised gelation, which in turn served as an injectable scaffold for stem cell growth. Later, the same research group exploited the use of PGMA- $b$-PHPMA diblock copolymer nanoworm gel in human Pluripotent Stem Cells (hPSCs) and human embryos. ${ }^{109}$ When cooled down from 37 to $5^{\circ} \mathrm{C}$, PGMA- $b$-PHPMA worm gels switched from worms to spheres, allowing for a reversible gel formation-destruction via an order-order transition, on which cells can be embedded and recovered. These worm gels showed a good aptitude at mimicking natural mammalian mucins and at forming an excellent 3D matrix to maintain hPSCs viability and pluripotency without any passaging for 14 days, as well as for possible short-term storage of hPSCs and human embryos without a need for cryopreservation.

\subsection{Enzymatically responsive systems}

Enzymes are key components in cell regulation, and enzymatic dysfunction or overexpression is associated with a number of pathological conditions, particularly in cancer. ${ }^{110-112}$ These dysfunctions or overexpressions can be exploited as a potential target in drug delivery designs. For instance, drugs can be leveraged when being exposed and degraded in a dysfunctional enzyme environment.

Although a majority of reported examples has been using RAFTPISA, other potential types of polymerisations can be of interest for the production of biodegradable and responsive polymer NPs. To address the challenge of high polymerisation temperatures and strictly oxygen- free conditions, ROMPISA has been introduced to synthesise particles with high control over the polymerisation process under aerobic conditions.

In a recent report, Wright et al. generated peptide-based NPs sensitive to the proteolytic enzyme thermolysin in aqueous media, via a one-pot method in open air upon ROMPISA at a high solid concentration $(10 \%) .^{71}$ Such enzyme-responsive poly(norbornene) block copolymers are key to a series of future applications where more enzymatically sensitive biodegradable materials can be prepared from the versatile and robust ROMPISA method.

Recently, vesicles made from the ROP of $\mathrm{N}$-carboxyanhydride (NCA)-induced self-assembly (NCA-PISA) have been suggested. The polymerisation of the L-phenylalanine NCA (L-Phe NCA) was initiated by methoxypolyethylene glycol amine $\left(\mathrm{PEG}_{45}-\mathrm{NH}_{2}\right)$ at $10^{\circ} \mathrm{C}$ without the need for degassing, using a $20 \%$ solid contents. ${ }^{70}$ The obtained vesicles were then degraded by trypsin over $96 \mathrm{~h}$.

\subsection{Dual photo- and pH-responsive systems}

The following formulation was proceeded upon a bifunctional responsiveness, endowing particles with the UV-induced crosslinking for enhanced colloidal stability under physiological conditions, while conferring $\mathrm{pH}$-triggered drug unloading in acidic microenvironments. In this contribution, two photosensitive monomers, 2-nitrobenzyl methacrylate (NBMA) and 7-(2methacryloyloxy-ethoxy)-4-methyl-coumarin (CMA) were employed as comonomers in a RAFT aqueous dispersion polymerisation with poly(N-(2-hydroxypropyl) methacrylamide (PHPMA) as a macro-CTA agent. This approach readily afforded well-defined polymeric nanostructures with four types of morphologies: spheres, worms, lamellae, and vesicles. ${ }^{113}$ Upon UV irradiation $(365 \mathrm{~nm})$, the NBMA moieties were cleaved and replaced with the ionised methyl methacrylate (MMA), inducing the hydrophobic-to-hydrophilic transition of the core-forming block; at the same time, the coumarin segments were dimerised to generate a core crosslinking, thus preventing the NP disassembly. DOX was loaded into the PHPMA- $b$-P(MAA-co-CMA) nano-objects via an electrostatic interaction between the $-\mathrm{NH}_{3}{ }^{+}$ammoniums on DOX and the $-\mathrm{COO}^{-}$ carboxylates on $\mathrm{P}(\mathrm{MAA}-\mathrm{co}-\mathrm{CMA})$ in water, enabling a relatively high drug loading of $24.9 \%$ wt. In vitro release revealed that DOX rapidly escaped from the nano-objects at $\mathrm{pH} 5.4$ in intracellular endosomes/lysosomes due to the protonation of the $-\mathrm{COO}^{-}$groups on PMAA, thus disrupting the electrostatic interactions between DOX and the hydrophobic P(MMA-co-CMA) core. In summary, the above formulation method has enabled polymeric NPs powerful 
dual responsive behaviours, exhibiting a robust photo-induced cross-linked core for greater stabilisation and on-demand DOX release in acidic intracellular microenvironment upon cellular uptake.

In a similar work, Pan et al. described the RAFT dispersion copolymerisation of DIPEMA and CMA in the presence of PHPMA as a macro-RAFT agent to produce in situ vesicles. ${ }^{114}$ Following irradiation with UV light at $365 \mathrm{~nm}$, the coumarin moieties anchored in the vesicle membrane underwent dimerisation to crosslink together, thus bringing an improved structural stabilisation to the polymeric vesicles. In addition, the presence of the tertiary amino groups on the DIPEMA segments of the membrane triggered a swelling-shrinking transition upon tuning the solution $\mathrm{pH}$ from acidic to basic. At $\mathrm{pH} 4$, the DIPEMA was protonated and shifted to a hydrophilic state, inducing the swelling of the core-forming block, thus enabling the pores of the membrane vesicles to open and facilitating the transmembrane traffic of substances. In acidic solutions, both the membrane (DIPEMA) and DOX were protonated, and the escape of DOX was inhibited due to electrostatic repulsion, although the membrane pores were large enough for DOX to get through. In contrast to DOX, fluorescein was effectively released after loading through the vesicle membrane due to a size of fluorescein smaller than those of the pores and the absence of electrostatic repulsions.

\subsection{Dual reductive- and $\mathrm{pH}-$ responsive systems}

To enhance the chemical versatility of membrane-forming block of the amphiphilic block copolymer vesicles, the dual in situ crosslinkers, $\mathrm{pH}$-responsive and a reductive-cleavable bond into the PISA-mediated copolymerisation was introduced. ${ }^{115}$ First, PEG-CPDB was chain-extended with comonomers including 2(diisopropylamino)-ethyl methacrylate (DIPEMA), and either methacrylamide multivinyl comonomer cysteaminebismethacrylamide (CBMA) or bis(2methacryloyloxyethyl) disulfide (DSDMA) using a RAFT-mediated dispersion polymerisation. It was indicated that the PISA of DIPEMA with the CBMA cross-linker imparted more robust crosslinked membranes. Rhodamine $B$ was used as a drug model and encapsulated into the polymeric vesicles. Owing to the dual stimuliresponsive character of the vesicles, the dye was rapidly unloaded

A In Situ Cross-linking in Polymerization-induced Self-assembly

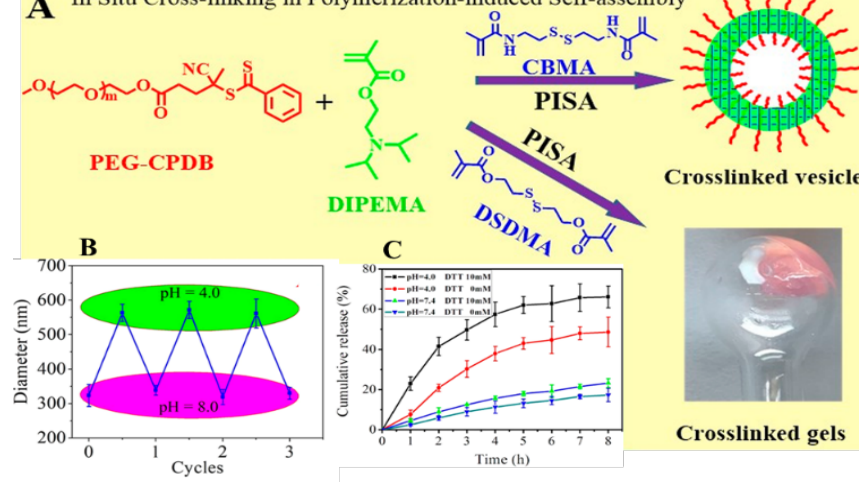

by the synergistic effect of DL-dithiothreitol (DTT) $(10 \mathrm{mM})$ and an acidic media at $\mathrm{pH} 4$ mimicking intracellular conditions. At acidic pHs, DIPEMA was protonated and switched the system to hydrophilic, vesicles thus swollen, and the dye was conclusively liberated. On the other hand, drug release was also extraaugmented due to the DTT-induced cleavage of disulfide cross-links in the acidic aqueous medium. This study demonstrated a straightforward one-pot strategy of the crosslinked membrane, self- assembly and drug encapsulation, endowing enhanced structural integrity and stabilisation to the vesicle membrane as well as an improved intracellular drug release (Fig 10).

\section{Outlooks and Future Challenges}

The present review discussed the latest exciting studies describing the design and formulation of in situ stimuli-responsive NPs via mostly RAFT-PISA and a few ROP/ROMP-PISA methods, in order to achieve on-demand drug release while favouring a simple and effortless (vraiment ?) synthetic approach. The distinctive heterogeneity of diseased body areas and their associated pathogenic conditions such as a higher reduction potential, the presence of ROS and/or low pHs have long become potential targets for the development of drug delivery systems that are responsive to these specific factors and enable drug unloading at the appropriate sites of action. The use of PISA is of particular interest and widely recognised as a powerful technique to prepare self-assembled NPs in a one-pot synthetic pathway, with limited intermediate purification steps, while facilitating scalability and industrial demands as opposed to traditional methods. A range of PISA-prepared self-assemblies incorporating functional moieties have been highlighted that respond to pathogenically mimicking stimuli. For example, NP pH-responsiveness in order to release drugs could be accomplished by an hydrophobic-to-hydrophilic solubility transition of the core-forming block. This transition resulted from the incorporation of ionisable groups that, upon acidic pHs associated to the cell pathology, induced protonation and hydrophilicity of the internal fragments, leading to nanoobjects disassembling and drugs being released.

Fig 10. (A) In situ crosslinking in PISA to prepare crosslinked PEGCPDB-b-P(DIPEMA90-co-CBMA10) membrane vesicles (D90C10); (B) Reversible changes in the diameter of $\mathrm{D} 90 \mathrm{C} 10$ vesicles upon switching solution $\mathrm{pH}$ between 4.0 and 8.0. (C) Cumulative release profiles of Rhodamine B from D90C10 vesicles under different conditions. Reprinted with permission from Ref ${ }^{115}$. Copyright 2019 American Chemical Society.

The nano-structural dissociation arising fom the solubility transition was also observed under ROS-abundant conditions, whereby the hydrophobic thioether moieties in the core were oxidised to hydrophilic sulfones, disintegrating the core-shell structures. Micelles cross-linked via disulfide bonds displayed an enhanced colloidal stability under normal cell conditions; however, in the presence of reductive agents, they disintegrated to liberate the internal payloads owing to the cleavage of reductive-sensitive disulfide bonds. On the other hand, thermal responsiveness of spherical micelles triggered drug release upon temperature changes because of the morphological evolutions. Dual triggers such as $\mathrm{pH}$-and-reduction or $\mathrm{pH}$-and-light stimuli combinations synergistically increased the sensitivity of particles for an enhanced drug release efficiency.

Although PISA research has vigorously been growing and displaying some great promises to overcome the drawbacks of traditional solution self-assembly techniques, many challenges remain to be addressed. One of the major bottlenecks preventing the expansion of PISA into more biomaterials applications are the relatively high temperatures and the absolute oxygen-free environments required by radical polymerisations, which can be challenging to achieve and hamper the final desired reaction yields. Most of the existing 
(meth)acrylate-based polymers are also difficult to degrade in biological media resulting in some increased accumulation and toxicity. Fortunately, some of these issues can be addressed using ROMP or ROP methods, involving the use of new and biodegradable monomers to synthesise self-assembled objects under mild conditions, or by using UV initiation to avoid the need of high reaction temperatures. These approaches are also of great benefit to the encapsulation of fragile therapeutic drugs or biomolecules such as peptides, antibodies, or DNA/RNA, thus avoiding their denaturation or degradation while retaining the structural integrity of self-assembly. Also, currently the majority of PISA formulation for drug delivery and biomaterials has been carried out based on RAFT polymerisations, some on ROP or ROMP. Hence there is a need to exploit other types of living radical polymerisations such as nitroxide-mediated polymerisations (NMP), ${ }^{116}$ iodine-mediated polymerisations (ITP), ${ }^{117}$ and sulfur-free RAFT ${ }^{118,119}$ for potentially preparing less toxic polymers. Though atom transfer radical polymerization (ATRP) has been extensively adopted to synthesise PISA-derived nano-objects, ${ }^{120,121}$ its application in drug delivery is very limited due to the use of cytotoxic copper catalysts during the polymerisation.

Also, it is of importance to be able to design multifunctional PISAmade particles that can load multiple therapeutic agents and/or diagnostic probes while being conjugated with targeting ligands for achieving enhanced site-specific drug delivery as well as precisely controlled drug release on demand. Additionally, more attempts are required to investigate the behaviour of these PISA-prepared NPs in delicate (complex ?) 3D cell culture models as well as in vivo, in order to rapidly accelerate translations to clinical studies. In principle, a formulation method at the hint of future interest should also display reasonable drug loading and increased cellular uptake or trafficking, and PISA has proven itself as an ideal candidate. Attempts have been made to apply external stimuli to impart higher control over sizes and morphologies of PISA-prepared NPs. Interestingly, light has been used to enable spatiotemporal control of initiation, ${ }^{122}$ cross-linking, ${ }^{113}$ or modification of the hydrophobic/hydrophilic block ratio. ${ }^{123}$

Nevertheless, while it is critical to carry out PISA in aqueous media for medical purposes, it can limit the ability of encapsulating hydrophobic drugs, especially via physical entrapment. Probably, the use of prodrug monomers as aforementioned could potentially provide a solution by which drugs can be conjugated to monomers or macro-CTAs prior to/during the PISA process via stimuliresponsive linkages, thus both increasing loading efficiency whilst yielding local release. Also, the question has been raised about whether particle shapes may have an impact on the resulting loading efficiency and cell internalisation since there have not been many reports addressing this issue. Hence more attention should definitely be drawn to this critical aspect. In the meanwhile, hydrophilic drugs can be easily compartmentalised into PISAgenerated NPs, particularly in vesicles, imparting the protection of internal cargos from a degradation by biological media. This aspect could be very useful for the preparation of less invasive oral formulation where drugs can be protected from the extreme conditions of the digestive and intestinal tracks before reaching the disease areas. Finally, the majority of PISA-related work up to now has been focusing on anticancer drug delivery while it is very likely that the same methodology could be applied to other therapeutic treatments, such as diabetes, and cardiovascular or infectious diseases.
Until the end, beyond these challenges, it is widely believed that PISA will continue to thrive and rapidly expedite (expand ?), building on our growing understanding of synthetic parameters and formulation strategies. To date, PISA has successfully enabled the self-assembly, drug loading and incorporation of stimuli-responsive functionalities all-in-one-pot synthetic approach. In a very soon future, one believes the rapid mature of PISA can instil more important aspects of the talented drug delivery into a selfassembled nano-object, thus furthering a range of therapeutic values to the ultimate translation from the bench to the bedside (???).

\section{Conflicts of interest}

There are no conflicts to declare.

\section{Acknowledgements}

The authors gratefully acknowledge the financial support of the Ministère de l'Enseignement supérieur, de la Recherche et de I'Innovation (MESRI) and Université Paris-Est Créteil. HP and BC acknowledge Jacques Penelle for his moral support throughout this work. 
Table 1. Summary of block copolymers, Stimulus/Stimuli, Drug/Payload and Morphology of nano-objects

\begin{tabular}{|c|c|c|c|c|}
\hline Block copolymer & Stimulus/Stimuli & Drug/Payload & Morphology & Ref. \\
\hline POEGMA- $b-\mathrm{P}(\mathrm{ST}-\mathrm{co}-\mathrm{VBA})$ & Acidic $\mathrm{pH}$ & Doxorubicin (DOX) & $\begin{array}{l}\text { Spheres, Worms, Rods, } \\
\text { Vesicles }\end{array}$ & 75 \\
\hline PDMAEMA & Acidic $\mathrm{pH}$ & DOX & $\begin{array}{l}\text { Spheres, Rods, Wires, } \\
\text { Vesicles }\end{array}$ & 76 \\
\hline $\mathrm{P}\left(\mathrm{HPMA}_{300}\right.$-stat-GlyMARh 1$)-\mathrm{PDPA}_{52}$ & Acidic $\mathrm{pH}$ & Rhodamine B & Vesicles & 77 \\
\hline PEG- $b-\mathrm{P}\left(\mathrm{MEO}_{2} \mathrm{MA}-c o-\mathrm{CPTM}\right)$ & GSH $10 \mathrm{mM}$ & Camptothecin (CPT) & Spheres & 85 \\
\hline P(CPTM-co-NIRM)- $b$-P(HPMA-co-EDGMA) & GSH $10 \mathrm{mM}$ & BODIPY & Spheres & 86 \\
\hline $\mathrm{PEG}_{113}-b$-PPFMA & GSH $10 \mathrm{mM}$ & None & Spheres & 87 \\
\hline $\mathrm{P}(\mathrm{NAM}-b-\mathrm{NAT})$ & $\mathrm{H}_{2} \mathrm{O}_{2} 10 \mathrm{mM}$ & Nile red & Spheres & 96 \\
\hline mPEG- $b$-P(HPMA-Co-DMAEMA) & $\mathrm{CO}_{2}$ & $\mathrm{SiO}_{2} \mathrm{NPs}$ & Vesicles & 104 \\
\hline PEGMA- $b$-P(HPMA-co-DMAEMA) & $\mathrm{CO}_{2}$ & BSA & Vesicles & 105 \\
\hline PGMA- $b$-P(HPMA-co-DMAEMA-co-AMA) & $\mathrm{CO}_{2}$ & $\begin{array}{l}\text { Methylene blue (MB)/ } \\
\mathrm{SiO}_{2} \mathrm{NPs}\end{array}$ & Vesicles & 106 \\
\hline PGMA- $b$-PHPMA & Temperature & $\mathrm{SiO}_{2} \mathrm{NPS}$ & Vesicles & 63 \\
\hline PHPMA- $b-\mathrm{P}(\mathrm{NBMA}-c o-\mathrm{CMA})$ & UV and acidic $\mathrm{pH}$ & DOX & $\begin{array}{l}\text { Spheres, Nanorods, } \\
\text { Lamellae, Vesicles }\end{array}$ & 113 \\
\hline PHPMA- $b$-P(DIPEMA-co-CMA) & UV and acidic $\mathrm{pH}$ & AuNPs/DOX/Fluorescein & Vesicles & 114 \\
\hline PEG-CPDB- $b$-P(DIPEMA-co-CBMA) & $\begin{array}{l}\text { Reductive (DTT) and acidic } \\
\mathrm{pH}\end{array}$ & Rhodamine B & Vesicles & 115 \\
\hline
\end{tabular}




\section{References}

A. K. Pearce and R. K. O'Reilly, Bioconjug. Chem., 2019, 30, 2300-2311.

2 V. Taresco, C. Alexander, N. Singh and A. K. Pearce, Adv. Ther., 2018, 1, 1800030.

3 P. Couvreur and C. Vauthier, Pharm. Res., 2006, 23, 14171450.

4 M. Karimi, A. Ghasemi, P. Sahandi Zangabad, R. Rahighi, S. M. Moosavi Basri, H. Mirshekari, M. Amiri, Z. Shafaei Pishabad, A. Aslani, M. Bozorgomid, D. Ghosh, A. Beyzavi, A. Vaseghi, A. R. Aref, L. Haghani, S. Bahrami and M. R. Hamblin, Chem. Soc. Rev., 2016, 45, 1457-1501. Z. S. Al-Ahmady, W. T. Al-Jamal, J. V. Bossche, T. T. Bui, A. F. Drake, A. J. Mason and K. Kostarelos, ACS Nano, 2012, 6, 9335-9346. S. Mura, J. Nicolas and P. Couvreur, Nat. Mater., 2013, 12, 991-1003.

J. V. John, C. W. Chung, R. P. Johnson, Y. II Jeong, K. D. Chung, D. H. Kang, H. Suh, H. Chen and I. Kim, Biomacromolecules, 2016, 17, 20-31. M. Liu, H. Du, W. Zhang and G. Zhai, Mater. Sci. Eng. C, 2017, 71, 1267-1280.

9 Z. Li, N. Song and Y. W. Yang, Matter, 2019, 1, 345-368.

10 G. Battistelli, A. Cantelli, G. Guidetti, J. Manzi and M. Montalti, Wiley Interdiscip. Rev. Nanomedicine Nanobiotechnology, 2016, 8, 139-150.

11 D. Shenoy, S. Little, R. Langer and M. Amiji, Pharm. Res. 2005, 22, 2107-2114. M. Elsabahy and K. L. Wooley, Chem. Soc. Rev., 2012, 41, 2545-2561.

13 A. Kumari, S. K. Yadav and S. C. Yadav, Colloids Surfaces B Biointerfaces, 2010, 75, 1-18.

14 K. S. Soppimath, T. M. Aminabhavi, A. R. Kulkarni and W. E. Rudzinski, J. Control. Release, 2001, 70, 1-20. S. Guragain, B. P. Bastakoti, V. Malgras, K. Nakashima and Y. Yamauchi, Chem. - A Eur. J., 2015, 21, 13164-13174. F. Jiang, S. Chen, Z. Cao and G. Wang, Polymer (Guildf)., 2016, 83, 85-91. N. Song, W. Liu, Q. Tu, R. Liu, Y. Zhang and J. Wang, Colloids Surfaces B Biointerfaces, 2011, 87, 454-463. T. Thambi, V. G. Deepagan, H. Y. Yoon, H. S. Han, S. H. Kim, S. Son, D. G. Jo, C. H. Ahn, Y. D. Suh, K. Kim, I. Chan Kwon, D. S. Lee and J. H. Park, Biomaterials, 2014, 35, 1735-1743. C. I. C. Crucho, ChemMedChem, 2015, 10, 24-38. A. W. Jackson and D. A. Fulton, Polym. Chem., 2013, 4, 3145. H. Phan, R. I. Minut, P. McCrorie, C. Vasey, R. R. Larder, E. Krumins, M. Marlow, R. Rahman, C. Alexander, V. Taresco and A. K. Pearce, J. Polym. Sci. Part A Polym. Chem., 2019, 57, 1801-1810.

P. Alexandridis and B. Lindman, Amphiphilic Block Copolymers: Self-Assembly and Applications, 2000. E. Batrakova, S. Lee, S. Li, A. Venne, V. Alakhov and A. Kabanov, Pharm. Res., 1999, 16, 1373-1379. Y. Yang, D. Pan, K. Luo, L. Li and Z. Gu, Biomaterials, 2013, 34, 8430-8443. X. B. Xiong, A. Falamarzian, S. M. Garg and A. Lavasanifar, in Journal of Controlled Release, Elsevier, 2011, vol. 155, pp. 248-261.

V. S. Trubetskoy, Adv. Drug Deliv. Rev., 1999, 37, 81-88. Y. Mai and A. Eisenberg, Chem. Soc. Rev., 2012, 41, 5969-
5985.

R. Raveendran, K. M. Mullen, R. M. Wellard, C. P. Sharma, R. Hoogenboom and T. R. Dargaville, Eur. Polym. J., 2017, 93, 682-694

S. J. T. Rezaei, M. R. Nabid, H. Niknejad and A. A. Entezami, Int. J. Pharm., 2012, 437, 70-79.

F. Xu, H. Li, Y. L. Luo and W. Tang, ACS Appl. Mater. Interfaces, 2017, 9, 5181-5192.

L. Zhang, H. Shen and A. Eisenberg, Macromolecules, 1997, 30, 1001-1011.

S. Yadav, A. K. Sharma and P. Kumar, Front. Bioeng. Biotechnol., 2020, 8, 127.

D. E. Discher and A. Eisenberg, Science (80-. )., 2002, 297, 967-973.

C. J. Ferguson, R. J. Hughes, B. T. T. Pham, B. S. Hawkett, R. G. Gilbert, A. K. Serelis and C. H. Such, Macromolecules, 2002, 35, 9243-9245.

N. P. Truong, J. F. Quinn, M. V Dussert, N. B. T. Sousa, M. R. Whittaker and T. P. Davis, ACS Macro Lett., 2015, 4, 381386.

X. Zhang, S. Boissé, W. Zhang, P. Beaunier, F. D'Agosto, J. Rieger and B. Charleux, Macromolecules, 2011, 44, 41494158.

B. Charleux, G. Delaittre, J. Rieger and F. D. Agosto, , DOI:10.1021/ma300713f

J. C. Foster, S. Varlas, B. Couturaud, J. R. Jones, R. Keogh, R. T. Mathers and R. K. O'Reilly, Angew. Chemie - Int. Ed., 2018, 57, 15733-15737.

M. Semsarilar, I. Canton and V. Ladmiral, in Methods in Molecular Biology, Humana Press Inc., 2016, vol. 1367, pp. 89-108.

F. D'Agosto, J. Rieger and M. Lansalot, Angew. Chemie - Int. Ed., 2020, 59, 8368-8392.

C. Liu, C.-Y. Hong and C.-Y. Pan, Polym. Chem., 2020, 11, 3673-3689.

D. Ikkene, A. A. Arteni, M. Ouldali, J.-L. Six and K. Ferji, Polym. Chem., , DOI:10.1039/DOPY00407C.

Y. Pei, A. B. Lowe and P. J. Roth, Macromol. Rapid Commun., 2017, 38, 1-14.

S. Y. Khor, J. F. Quinn, M. R. Whittaker, N. P. Truong and T. P. Davis, Macromol. Rapid Commun., 2019, 40, 1-22. J. C. Foster, S. Varlas, B. Couturaud, Z. Coe and R. K. O'Reilly, J. Am. Chem. Soc., 2019, 141, 2742-2753. J. Rieger, Macromol. Rapid Commun., 2015, 36, 14581471.

X. Wang and Z. An, Macromol. Rapid Commun., 2019, 40, 1800325

D. Le, D. Keller and G. Delaittre, Macromol. Rapid Commun., 2019, 40, 1-21.

W. J. Zhang, C. Y. Hong and C. Y. Pan, Macromol. Rapid Commun., 2019, 40, 1-10. Macro Lett., 2019, 8, 1029-1054.

Y. Zhao, Y. Wang, F. Ran, Y. Cui, C. Liu, Q. Zhao, Y. Gao, D. Wang and S. Wang, Sci. Rep., 2017, 7, 1-11.

J. Zhao, H. Lu, S. Wong, M. Lu, P. Xiao and M. H. Stenzel, Polym. Chem., 2017, 8, 3317-3326.

53 A. B. Jindal, Int. J. Pharm., 2017, 532, 450-465.

54 S. Salatin, S. Maleki Dizaj and A. Yari Khosroushahi, Cell Biol. Int., 2015, 39, 881-890.

55 Nanomedicine, 2014, 9, 121-134. 
R. Toy, E. Hayden, C. Shoup, H. Baskaran and E. Karathanasis, Nanotechnology, , DOI:10.1088/09574484/22/11/115101. Cigler, H. C. Chang, G. Lin, R. Liu and Q. Li, Sci. Rep., 2014, 4, 1-9. W. Zhao, H. T. Ta, C. Zhang and A. K. Whittaker, Biomacromolecules, 2017, 18, 1145-1156. H. Soo Choi, W. Liu, P. Misra, E. Tanaka, J. P. Zimmer, B. Itty Ipe, M. G. Bawendi and J. V. Frangioni, Nat. Biotechnol., 2007, 25, 1165-1170.

. Bertrand, P. Grenier, M. Mahmoudi, E. M. Lima, E. A. Appel, F. Dormont, J. M. Lim, R. Karnik, R. Langer and O. C. Farokhzad, Nat. Commun., , DOI:10.1038/s41467-01700600-w. C. Fang, B. Shi, Y. Y. Pei, M. H. Hong, J. Wu and H. Z. Chen, Eur. J. Pharm. Sci., 2006, 27, 27-36. . Engström, H. Asem, H. Brismar, Y. Zhang, M. Malkoch and E. Malmström, Macromol. Chem. Phys., , DOI:10.1002/macp.201900443.

C. J. Mable, R. R. Gibson, S. Prevost, B. E. McKenzie, O. O. Mykhaylyk and S. P. Armes, J. Am. Chem. Soc., 2015, 137, 16098-16108.

M. J. Derry, L. A. Fielding and S. P. Armes, Prog. Polym. Sci., 2016, 52, 1-18.

B. Karagoz, C. Boyer and T. P. Davis, Macromol. Rapid Commun., 2014, 35, 417-421.

F. L. Hatton, J. R. Lovett and S. P. Armes, Polym. Chem., 2017, 8, 4856-4868.

J. Tan, Y. Bai, X. Zhang, C. Huang and D. Liu, 1434-1440.

J. Siirilä, S. Häkkinen and H. Tenhu, Polym. Chem., 2019, 10, 766-775.

J. Lesagedelahaye, X. Zhang, I. Chaduc, F. Brunel, M. Lansalot and F. D'Agosto, Angew. Chemie - Int. Ed., 2016, 55, 3739-3743.

J. Jiang, X. Zhang, Z. Fan and J. Du, ACS Macro Lett., 2019, 8, 1216-1221.

D. B. Wright, M. P. Thompson, M. A. Touve, A. S. Carlini and N. C. Gianneschi, Macromol. Rapid Commun., 2019, 40, 1800467.

K. Bauri, A. Narayanan, U. Haldar and P. De, Polym. Chem., 2015, 6, 6152-6162.

A. Punnia-Moorthy, J. Oral Pathol. Med., 1987, 16, 36-44. G. Hao, Z. P. Xu and L. Li, RSC Adv., 2018, 8, 22182-22192.

B. Karagoz, L. Esser, H. T. Duong, J. S. Basuki, C. Boyer and T. P. Davis, Polym. Chem., 2014, 5, 350-355.

L. Qiu, C. Xu, F. Zhong, C. Hong and C. Pan, ACS Appl. Mater. Interfaces, 2016, 8, 18347-18359.

C. J. Mable, I. Canton, O. O. Mykhaylyk, B. Ustbas Gul, P. Chambon, E. Themistou and S. P. Armes, Chem. Sci., 2019, 10, 4811-4821.

D. B. Wright, M. T. Proetto, M. A. Touve and N. C.

Gianneschi, Polym. Chem., 2019, 10, 2996-3000.

M. P. Gamcsik, M. S. Kasibhatla, S. D. Teeter and O. M. Colvin, Biomarkers, 2012, 17, 671-691.

H. J. Forman, H. Zhang and A. Rinna, Mol. Aspects Med. 2009, 30, 1-12.

J. M. Estrela, A. Ortega and E. Obrador, Crit. Rev. Clin. Lab. Sci., 2006, 43, 143-181.

R. Bakalova, Z. Zhelev, I. Aoki and T. Saga, Clin. Cancer Res. 2013, 19, 2503-2517.

Z. Li, M. Wu, H. Bai, X. Liu and G. Tang, Chem. Commun.,
2018, 54, 13127-13130.

J. Zhen, S. Tian, Q. Liu, C. Zheng, Z. Zhang, Y. Ding, Y. An, Y. Liu and L. Shi, Biomater. Sci., 2019, 7, 2986-2995.

W. Zhang, C. Hong and C. Pan, Biomacromolecules, 2016, 17, 2992-2999.

C. Tian, J. Niu, X. Wei, Y. Xu, L. Zhang, Z. Cheng and X. Zhu, Nanoscale, 2018, 10, 10277-10287.

B. Couturaud, P. G. Georgiou, S. Varlas, J. R. Jones, M. C. Arno, J. C. Foster and R. K. O'Reilly, Macromol. Rapid Commun., 2019, 40, 1-6.

X. Zhao, M. Chen, W. G. Zhang, C. H. Wang, F. Wang, Y. Z. You, W. J. Zhang and C. Y. Hong, Macromol. Rapid Commun., 2020, 41, 2000260.

P. Jia, C. Dai, P. Cao, D. Sun, R. Ouyang and Y. Miao, RSC Adv., 2020, 10, 7740-7750.

K. Apel and H. Hirt, Annu. Rev. Plant Biol., 2004, 55, 373399.

L. Lin, H. Gong, R. Li, J. Huang, M. Cai, T. Lan, W. Huang, Y. Guo, Z. Zhou, Y. An, Z. Chen, L. Liang, Y. Wang, X. Shuai and K. Zhu, Adv. Sci., 2020, 7, 1903138.

X. Xia, X. Yang, P. Huang and D. Yan, ACS Appl. Mater. Interfaces, 2020, 12, 18301-18308.

J. Shi, Z. Chen, B. Wang, L. Wang, T. Lu and Z. Zhang, ACS Appl. Mater. Interfaces, 2015, 7, 28554-28565.

Y. Yao, H. Zhang, Z. Wang, J. Ding, S. Wang, B. Huang, S. Ke and C. Gao, J. Mater. Chem. B, 2019, 7, 5019-5037. Y. Na, J. S. Lee, J. Woo, S. Ahn, E. Lee, W. II Choi and D. Sung, J. Mater. Chem. B, 2020, 8, 1906-1913. F. H. Sobotta, F. Hausig, D. O. Harz, S. Hoeppener, U. S. Schubert and J. C. Brendel, Polym. Chem., 2018, 9, 15931602.

A. Darabi, P. G. Jessop and M. F. Cunningham, Chem. Soc. Rev., 2016, 45, 4391-4436.

Q. Yan, J. Wang, Y. Yin and J. Yuan, Angew. Chemie - Int. Ed., 2013, 52, 5070-5073.

J. Gutknecht, M. A. Bisson and F. C. Tosteson, J. Gen. Physiol., 1977, 69, 779-794.

Q. Yan, R. Zhou, C. Fu, H. Zhang, Y. Yin and J. Yuan, Angew. Chemie - Int. Ed., 2011, 50, 4923-4927.

Q. Yan and Y. Zhao, Chem. Commun., 2014, 50, 1163111641.

H. Wu, F. Li, S. Wang, J. Lu, J. Li, Y. Du, X. Sun, X. Chen, J. Gao and D. Ling, Biomaterials, 2018, 151, 66-77.

J. H. Kim, H. Kim, Y. Choi, D. S. Lee, J. Kim and G. R. Yi, ACS Appl. Mater. Interfaces, 2017, 9, 31469-31477.

J. Tan, D. Liu, X. Zhang, C. Huang, J. He, Q. Xu, X. Li and L. Zhang, RSC Adv., 2017, 7, 23114-23121.

J. Tan, X. Zhang, D. Liu, Y. Bai, C. Huang, X. Li and L. Zhang, Macromol. Rapid Commun., 2017, 38, 1-8.

L. Yu, Y. Zhang, X. Dai, Q. Xu, L. Zhang and J. Tan, Chem. Commun., 2019, 55, 11920-11923. and L. Leibler, Nature, 2014, 505, 382-385.

A. Blanazs, R. Verber, O. O. Mykhaylyk, A. J. Ryan, J. Z. Heath, C. W. I. Douglas and S. P. Armes, J. Am. Chem. Soc., 2012, 134, 9741-9748.

I. Canton, N. J. Warren, A. Chahal, K. Amps, A. Wood, R. Weightman, E. Wang, H. Moore and S. P. Armes, ACS Cent. Sci., 2016, 2, 65-74.

C. E. Callmann, C. V. Barback, M. P. Thompson, D. J. Hall, R. F. Mattrey and N. C. Gianneschi, Adv. Mater., 2015, 27, 4611-4615. 
111 J. E. Ghadiali and M. M. Stevens, Adv. Mater., 2008, 20, 4359-4363.

112 M. Li, G. Zhao, W.-K. Su and Q. Shuai, Front. Chem., 2020, 8, 647 .

113 W. J. Zhang, C. Y. Hong and C. Y. Pan, Biomacromolecules, 2017, 18, 1210-1217.

114 W. J. Zhang, C. Y. Hong and C. Y. Pan, ACS Appl. Mater. Interfaces, 2017, 9, 15086-15095.

115 M. Chen, J. W. Li, W. J. Zhang, C. Y. Hong and C. Y. Pan, Macromolecules, 2019, 52, 1140-1149.

116 E. Groison, S. Brusseau, F. D’Agosto, S. Magnet, R. Inoubli, L. Couvreur and B. Charleux, ACS Macro Lett., 2012, 1, 4751.

117 S. Shanmugam, J. Xu and C. Boyer, J. Am. Chem. Soc., 2015, 137, 9174-9185.

118 A. Lotierzo, R. M. Schofield and S. A. F. Bon, ACS Macro Lett., 2017, 6, 1438-1443.

119 J. Sarkar, L. Xiao, A. W. Jackson, A. M. Van Herk and A. Goto, Polym. Chem., 2018, 9, 4900-4907.

120 J. Wang, Z. Wu, G. Wang and K. Matyjaszewski, Macromol. Rapid Commun., , DOI:10.1002/marc.201800332.

121 Y. Wang, G. Han, W. Duan and W. Zhang, Macromol. Rapid Commun., 2019, 40, 1800140.

122 J. Yeow and C. Boyer, Adv. Sci., 2017, 4.

123 Q. Ye, M. Huo, M. Zeng, L. Liu, L. Peng, X. Wang and J. Yuan, Macromolecules, 2018, 51, 3308-3314. 